\title{
Effect of dehydration heat exposure on thoracic aorta reactivity in rats
}

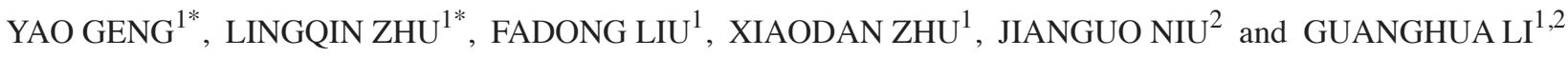 \\ ${ }^{1}$ Department of Physiology, School of Basic Medical Science, Ningxia Medical University; \\ ${ }^{2}$ Ningxia Key Laboratory of Cranial Cerebral Diseases, Yinchuan, Ningxia 750004, P.R. China
}

Received June 3, 2016; Accepted September 13, 2016

DOI: 10.3892/br.2016.760

\begin{abstract}
The aim of the present study was to investigate the effect of one week dehydration heat exposure on thoracic aorta reactivity in rats. Eighteen Male Sprague-Dawley rats were randomly divided into 3 groups ( $n=6$ each group): Control group (CN), heat exposure group ( $\mathrm{HE})$, dehydration heat exposure group (DHE). The $\mathrm{CN}$ group was exposed to a room temperature of $24^{\circ} \mathrm{C}$, while the $\mathrm{HE}$ and DHE groups were exposed to a heat temperature of $32^{\circ} \mathrm{C}$. After 7 days of heat exposure, the heart rate and blood pressure of the rats were measured, and the noradrenaline (NA)-induced contraction on the aorta rings was measured by tension recording. The average contents of malondialdehyde (MDA) and superoxide dismutase (SOD) in serum were detected using ELISA. The expression of apoptotic genes in the thoracic aorta was measured using RT-PCR. Compared with $\mathrm{CN}$, the heart rate in the HE and DHE groups had a tendency to become retarded, but there was no significant difference $(\mathrm{P}>0.05)$. In the HE group, the systolic blood pressure (SBP), diastolic blood pressure (DBP) and mean arterial pressure (MAP) of the rats were significantly higher than that of the $\mathrm{CN}(\mathrm{P}<0.05)$. In the DHE group, the SBP of rats was significantly higher than that of the $\mathrm{CN}(\mathrm{P}<0.05)$, while the SBP, DBP, and MAP of the rats were decreased compared to the rats in the HE group, although there was no statistical significance $(\mathrm{P}>0.05)$. In the $\mathrm{HE}$ and $\mathrm{DHE}$ groups, the NA-induced contraction on the rats thoracic aorta ring was larger than that of the $\mathrm{CN}(\mathrm{P}<0.05)$, albeit there was no significant difference between the $\mathrm{HE}$ and DHE groups $(\mathrm{P}>0.05)$. The serum SOD content decreased in the HE and DHE groups, however, the reduction was significant only in the DHE group $(\mathrm{P}<0.05)$. The content
\end{abstract}

Correspondence to: Dr Guanghua Li, Ningxia Key Laboratory of Cranial Cerebral Diseases, 1160 Shengli Street, Yinchuan, Ningxia 750004, P.R. China

E-mail: ghlee0404@163.com

*Contributed equally

Key words: high temperature, blood vessel compliance, cell apoptosis of MDA in serum was significantly increased in the DHE group $(\mathrm{P}<0.05)$. The expression of BAX was significantly upregulated whereas $\mathrm{Bcl} 2$ expression was decreased in the DHE group $(\mathrm{P}<0.05)$. The results showed that a high temperature was harmful to the body, especially in the case of lack of food and water. Additionally, the heat exposure elevated blood pressure, and increased arterial reactivity, which were related to the elevated production of MDA, led to the impaired production of SOD, and an increase of cell apoptosis. These findings are useful to understand the influence of dehydrated heat exposure on the vascular function, and they provide certain theoretical and experimental guidance for protection under high temperature.

\section{Introduction}

High temperature has a severe influence on an individual's work, life and body, and it is easy for the individual to become exhausted, irritable and exasperation. A high temperature constitutes a risk factor for the occurrence of cerebrovascular, heart and respiratory diseases; consequently, the death rate increases correspondingly, particularly among the elderly (1-3). At present, the effect of a high temperature especially the dehydration of thermal on the physiological function of human, is lacking in terms of comprehensive knowledge and understanding.

On the basis of heat stress, if the water intake is stopped, the extracellular fluid is reduced more rapidly and becomes DHE (4). For some workers such as firefighters, the damage caused by high temperature is inevitable. Recent findings have shown that dehydrated heat exposure elevated blood pressure over a long period of time increased the viscera index of spleen, heart, thymus gland, hypothalamus and pituitary to alleviate the harmful effects of high temperature on the body (5). However, the physiopathological mechanism involved in dehydration heat exposure on the cardiovascular system remains unknown. Thus, the present study focused on whether the influence of dehydrated heat exposure on the function of thoracic aorta is useful to understand the altered function of blood vessels. This study discussed the influence of dehydrated heat exposure on the thoracic artery of rats by studying the blood pressure, arterial reactivity, superoxide dismutase (SOD) and malondialdehyde (MDA) serums and apoptotic function. 


\section{Materials and methods}

Animals and heat exposure protocol. Eighteen male Sprague-Dawley rats, weighing 180-200 g, were purchased from the Laboratory Animal Center of Ningxia Medical University (Ningxia, China). The experimental procedures of the present study were approved by the Animal Ethics Committee of Ningxia Medical University and Use Committee, in accordance with the guidelines of the Council of the Physiological Society of China.

Eighteen rats were randomly divided into control group $(\mathrm{CN})$, heat exposure group (HE) and dehydration heat exposure group (DEF) ( $\mathrm{n}=6 /$ group). Rats in the $\mathrm{CN}$ group were fed at room temperature $\left(25 \pm 1^{\circ} \mathrm{C}\right)$ throughout the study and were provided with food and water ad libitum. Rats in the HE and DHE groups received a fixed $8 \mathrm{~h}$ (9:00-17:00) heat exposure process per day, and in the DHE group, the rats were fasted during the exposed time. Exposure was finished inside the artificial climate chamber with a temperature of $32^{\circ} \mathrm{C}$ (relative humidity of $60 \pm 5 \%$ ), after exposure, and the rats were kept at room temperature $\left(25 \pm 1^{\circ} \mathrm{C}\right)$. The heat exposure lasted for 7 days. Room temperature was $24.0 \pm 0.1^{\circ} \mathrm{C}$ and relative humidity was $54 \pm 5 \%$. The behavior of the animals was observed in the process of the whole experiment.

Heart rate and blood pressure. Heart rate and blood pressure were collected using blood pressure monitor (BP-2010A; Softron Beijing Biotechnology Co., Ltd., Beijing, China), and the data were obtained directly from the machine. After the heat exposure, we measured the heart rate and blood pressure from the rat tail.

Thoracic aortia reactivity. After anesthesia, the chest of the rats was immediately opened, and the thoracic aorta was removed and placed in a paraffin plate filled with physiological saline. Connective tissues were excised carefully, and vascular rings were made (4-5 $\mathrm{mm}$ wide). The vascular ring was quickly hung in the organization bath systems with the presentation of $10 \mathrm{ml}$ Krebs solution [ingredients (mmol/l): 5.6 glucose, $10 \mathrm{NaCl}$, $24.8 \mathrm{NaHCO}_{3}, 4.6 \mathrm{KCl}, 2.5 \mathrm{CaCl}_{2}$, as well as $1.2 \mathrm{MgSO}_{4}$ and $\mathrm{KH}_{2} \mathrm{PO}_{4}$, respectively]. The system was perfuse with $5 \% \mathrm{CO}_{2}$ and $95 \% \mathrm{O}_{2}$ contiguously and maintained a constant temperature of $37^{\circ} \mathrm{C}$. Resting tension was adjusted to $1 \mathrm{~g}$, and the ring was balanced for 40 min with Krebs changed every $15 \mathrm{~min}$. The maximal contraction was induced by the addition of $60 \mathrm{mM} \mathrm{KCl}$. After resting tension was stabilized, the Krebs fluid was replaced and basal tension was returned to $1 \mathrm{~g}$, and cumulative concentrations of norepinephrine $\left(10^{-10}-10^{-5} \mathrm{M}\right)$ was added to the bath system. The rates of the vascular tension range induced by noradrenaline (NA) $\left(10^{-10}-10^{-5} \mathrm{M}\right)$ were expressed as percentages of the maximum contraction tension range $(100 \%)$ induced by $\mathrm{KCl}(60 \mathrm{mM})$.

Detection of the serum SOD and MDA. Blood was collected from the left atrium and centrifuged at 3,500 $\mathrm{x}$ g for $15 \mathrm{~min}$ and the serum was segregated for further detection. The serum SOD was detected using a commercially available sandwich ELISA kit (Chenglin Biotechnology, Beijing, China). The MDA was detected by TBA kit (Nanjing Jiancheng Bioengineering Research Institute, Jiangsu, China). All the detections were tested in accordance with the kit's specifications. Absorbance was read at $450 \mathrm{~nm}$ (Bio-Rad 680; Bio-Rad, Hercules, CA, USA). The quantity of SOD in the serum was estimated from a calibration curve.

Detection of Bcl-2 and BAX gene expression. Vascular thoracic aortas were quickly removed and placed into liquid nitrogen. Frozen samples were reserved at $-80^{\circ} \mathrm{C}$ for further analysis. The thoracic aorta $(50 \mathrm{mg})$ was homogenized using glass-Teflon ${ }^{\circledR}$. Total RNA was prepared using TRIzol ${ }^{\circledR}$ Reagent (Invitrogen, Thermo Fisher Scientific, Inc., Waltham, MA, USA) according to the manufacturer's instructions. Complementary DNA (cDNA) was synthesized with a First Strand cDNA Synthesis kit (Thermo Fisher Scientific, Inc., Beijing, China). RT-PCR was carried out using a Maxima SYBR-Green PCR kit (Thermo Fisher Scientific, Inc.) with indicated primers. After an initial $10 \mathrm{~min}$ at $95^{\circ} \mathrm{C}$, the PCR program was finished as follows: $95^{\circ} \mathrm{C}$ for $15 \mathrm{sec}, 60^{\circ} \mathrm{C}$ for $30 \mathrm{sec}$, and extension at $72^{\circ} \mathrm{C}$ for $30 \mathrm{sec}$, for 40 cycles. At the end of the reaction, melting curve analysis was performed to ensure the specificity of the reaction. $\beta$-actin was used as an internal control. Primers used for the PCR are shown in Table I.

Statistical analysis. Data were analyzed by SPSS, Inc. 21.0 (Chicago, IL, USA), and the results were presented as mean \pm SD. Statistical difference was evaluated using the t-test. $\mathrm{P}<0.05$ was considered to indicate a statistically significant difference.

\section{Results}

Behavior observation in rats. Before heat exposure, all the rats had good appetite, activity and agile reaction. Their fur was tight, clean and smooth. The rats were restless in the evening. During thermal exposure, the rats' limbs, nasal and testicular filled with blood, which was more obvious in the DHE group. Concerning mental state, the rats were tired and listless. Their surface fur was damp, the response to external stimuli was significantly reduced, and the rats hid their body in bedding material.

Measurement of heart rate and blood pressure. Compared with $\mathrm{CN}$, the heart rate in the HE and DHE groups was retarded, albeit there was no significant difference $(\mathrm{P}>0.05)$ (Fig. 1). In the HE group, SBP, DBP and the mean arterial pressure (MAP) of rats were significantly higher than the $\mathrm{CN}$ $(\mathrm{P}<0.05)$. In the DHE group, the SBP of rats was significantly higher than that of the $\mathrm{CN}(\mathrm{P}<0.05)$, albeit SBP, DBP and MAP of rats were lower than the rats in the HE group, although there was no statistical significance $(\mathrm{P}>0.05)$ (Fig. 2).

Thoracic aortia reactivity. In the $\mathrm{CN}$ and HE groups, NA induced a dose-dependent contraction on the aortic ring. However, the contractive response was stronger in the HE compared with the $\mathrm{CN}$ group $(\mathrm{P}<0.05)$, but there was no significant difference between the HE and DHE groups $(\mathrm{P}>0.05)$ (Fig. 3).

Detection of the serum SOD and MDA. The serum SOD in the DHE group was lower than that of the $\mathrm{CN}$ group 
Table I. Sequence of oligonucleotide primers.

\begin{tabular}{llccr}
\hline Gene & \multicolumn{1}{c}{ Sequence $\left(5^{\prime}->3^{\prime}\right)$} & bp & Tm/ ${ }^{\circ} \mathrm{C}$ & GenBank \\
\hline$B c 12$ & $\begin{array}{l}\text { Forward: AGCCTGAGAGCAACCGAAC } \\
\text { Reverse: AGCGACGAGAGAAGTCATCC }\end{array}$ & 159 & 60 & NM_016993 \\
Bax & $\begin{array}{l}\text { Forward: TTGCTACAGGGTTTCATCCAG } \\
\text { Reverse: TGTTGTTGTCCAGTTCATCG }\end{array}$ & 145 & 60 & NM_017059 \\
-actin & $\begin{array}{l}\text { Forward: CACCCGCGAGTACAACCTTC } \\
\text { Reverse: CCCATACCCACCATCACACC }\end{array}$ & 207 & 60 & NM_031144 \\
\hline
\end{tabular}

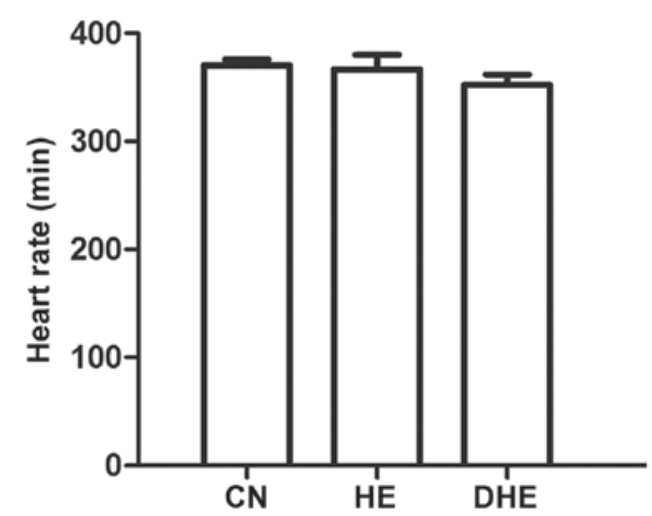

Figure 1. Effect of heat exposure on heart rate. Data are shown as mean $\pm \mathrm{SD}$, $\mathrm{n}=6$. $\mathrm{CN}$, control group; HE, heat exposure group; DHE, dehydration heat exposure.

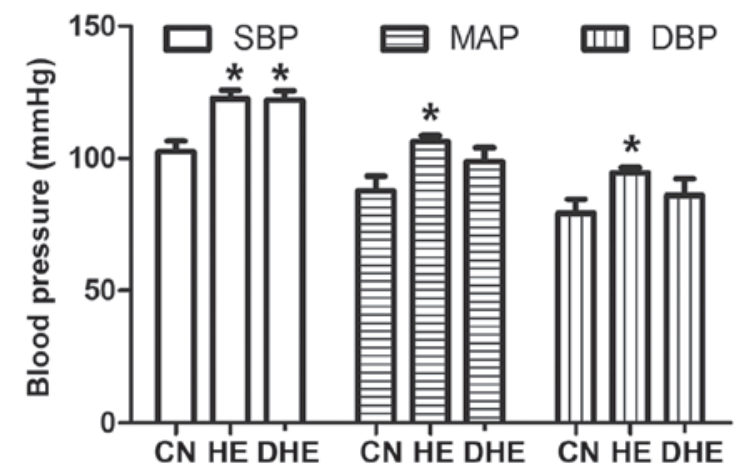

Figure 2. Effect of heat exposure on blood pressure. Data are shown as mean \pm standard deviation, $\mathrm{n}=6$. $\mathrm{CN}$, control group; HE, heat exposure group; DHE, dehydration heat exposure. ${ }^{*} \mathrm{P}<0.05$ compared with $\mathrm{CN}$.

$(\mathrm{P}<0.05)$ (Fig. 4), the level of MDA was significantly higher than that of the $\mathrm{CN}$ group $(\mathrm{P}<0.05)$ (Fig. 5). Compared with the CN and DHE groups, the serum SOD and MDA in the HE group was fluctuated but no significant difference was observed $(\mathrm{P}>0.05)$.

Detection of Bcl-2 and Bax. Compared with $\mathrm{CN}$, the expression of Bcl2 was significantly reduced $(\mathrm{P}<0.05)$ in the DEF group, while the Bax expression was increased $(\mathrm{P}<0.05)$. Compared with teh $\mathrm{CN}$ and DHE groups, the expression of $\mathrm{Bcl} 2$ and $\mathrm{Bax}$ in the HE group had no significant difference (P>0.05) (Fig. 6).

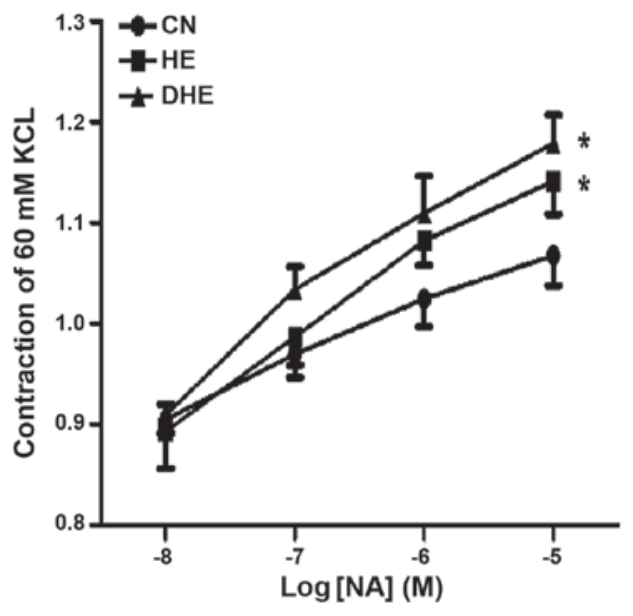

Figure 3. Effect of heat exposure on thoracic aortic reactivity. Data are shown as mean \pm standard deviation, $\mathrm{n}=6$. $\mathrm{CN}$, control group; HE, heat exposure group; DHE, dehydration heat exposure. ${ }^{*} \mathrm{P}<0.05$ compared with $\mathrm{CN}$.

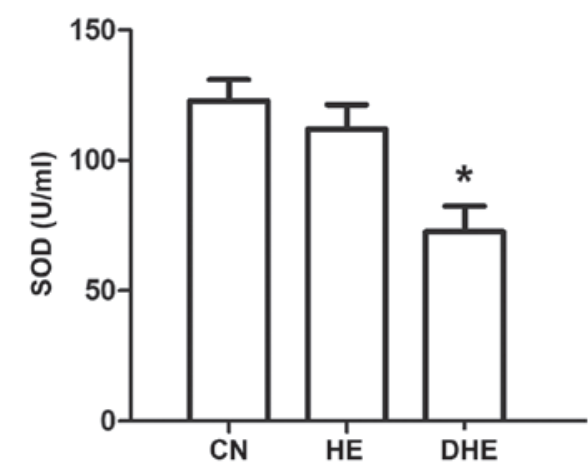

Figure 4. Effect of heat exposure on the level of SOD in serum of rats. Data are shown as mean $\pm \mathrm{SD}, \mathrm{n}=8$. $\mathrm{CN}$, control group; $\mathrm{HE}$, heat exposure group; DHE, dehydration heat exposure; SOD, superoxide dismutase. ${ }^{*} \mathrm{P}<0.05 \mathrm{com}-$ pared with $\mathrm{CN}$.

\section{Discussion}

The present study has demonstrated that heat stress without food and water changed the rats' existence, increased blood pressure and damaged blood vessel function.

Living or working in a high temperature environment leads to not adapting to adverse reactions (6). Consequently, a lack of food and water reduces an individual's ability to survive. In a high temperature environment, the evaporation of the skin becomes the main way of cooling the body, 


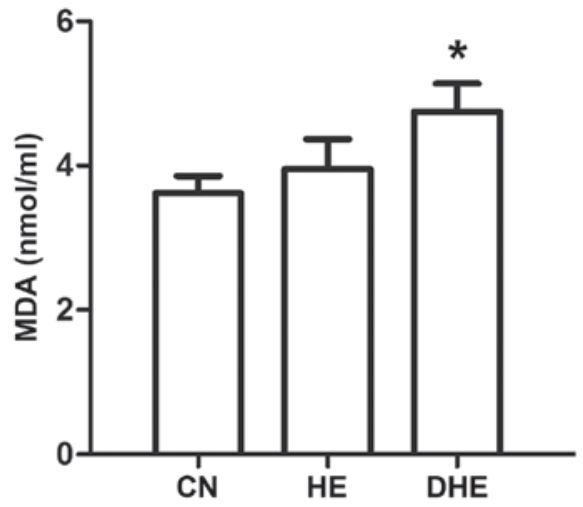

Figure 5. Effect of heat exposure on the level of MDA in serum of rats. Data are shown as mean \pm standard deviation, $\mathrm{n}=8$. $\mathrm{CN}$, control group; HE, heat exposure group; DHE, dehydration heat exposure; MDA malondialdehyde. ${ }^{*} \mathrm{P}<0.05$ compared with $\mathrm{CN}$.

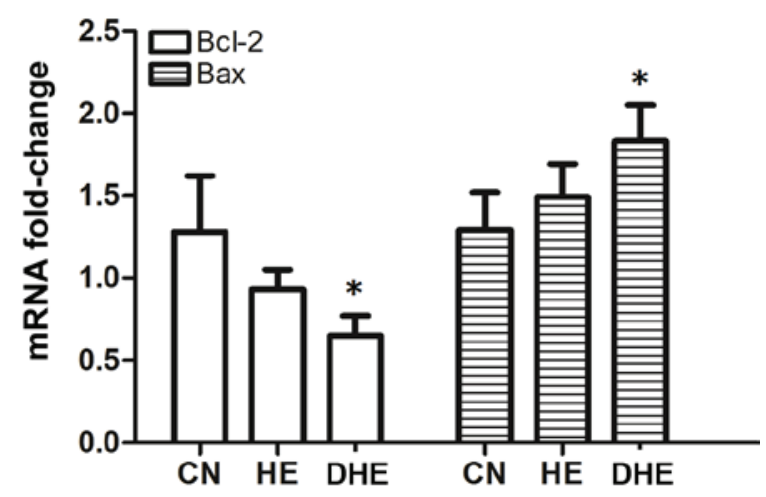

Figure 6. Effect of heat exposure on the expression of Bcl-2 and Bax. Data are shown as mean \pm standard deviation, $\mathrm{n}=8$. $\mathrm{CN}$, control group; HE, heat exposure group; DHE, dehydration heat exposure. ${ }^{~} \mathrm{P}<0.05$ compared with $\mathrm{CN}$.

but the high humidity limits the evaporation of perspiration. Under water shortage conditions, excessive loss of perspiration may damage the body's cardiovascular and thermoregulatory function (7-9). In response to the high temperature, the human body needs heat dissipation and expansion of body surface blood vessels, the amount of blood in the body surface increases, and the amount of blood supply for heart, cerebrovascular is relatively reduced, leading to cerebral ischemic and hypoxic reactions $(10,11)$. The experiment results showed that, with the increase of heat exposure intensity, the rat heart rate had a tendency to slow down. Overton et al found that hunger and high temperature were able to reduce the heart and metabolic rate (12). The study by Williams et al showed hunger and high temperature were associated with the gradual reduction of activity (13). These indicated that, under the condition of hunger and high temperature, the rats could reduce heat production by decreasing movement and the metabolic rate. In the HE group, SBP, DBP, MAP were significantly higher than that of $\mathrm{CN}$. The SBP of the rats in the DHE group was significantly higher than that of the $\mathrm{CN}$. These results showed that high temperature increased blood pressure.

Vascular reactivity is a basic and direct index that reflects the state of the artery blood vessel function (14). Enhanced contractive function is the main performance of damaged blood vessels $(15,16)$. The experiment results showed that the thoracic aorta reactivity in the HE and DHE groups was higher than that of the $\mathrm{CN}$ group. The environment of high temperature is harmful to blood vessels. However, it is difficult to find vascular injury; we investigated blood vessel function and attempted to identify the mechanism underlying vascular injury.

SOD is an important enzyme, which removes superoxide free radical from aerobic organisms and defense against the toxic effect of the oxygen-free radicals $(17,18)$. MDA is the oxidative product of polyunsaturated fat in biological membrane (19). Its level can reflect the degree of lipid peroxidation and free radicals attacking the body's cells. In this experiment, SOD decreased significantly whereas the MDA level was increased in the the DHE group. This result suggested that high temperature without food and water caused increase of endogenous oxygen free radicals. Therefore, enhanced oxidative stress damaged the vascular elasticity.

Cell apoptosis was induced by stress, such as free radicals, hypoxia and blood deficiency $(20,21)$. The present study focused on the function of $\mathrm{Bcl} 2$ and Bax in the regulation of apoptosis. In the process of cell apoptosis, members of the Bcl-2 family play a vital role. They have high homology, and share the conserved domain of $\mathrm{BH} 1, \mathrm{BH} 2, \mathrm{BH} 3$ and $\mathrm{BH} 4$. The $\mathrm{Bcl} 2$ family can be divided into two categories, one of which is anti-apoptotic, mainly containing Bcl2, Bcl-XL, Bcl-W, Mcl-1 and CED9. The other type promotes cell death, and mainly includes Bax, Bak, Bcl-XS, Bad, Bik and Bid. Increased Bax promotes cell apoptosis, whereas increased $\mathrm{Bcl} 2$ inhibits cell apoptosis (22). In this experiment, the expression of $\mathrm{Bcl} 2$ was significantly reduced in the DHE group, while Bax was increased. The present study has demonstrated that under the condition of dehydration heat exposure, the expression of Bax was markedly elevated, whereas Bcl2 was reduced. These results suggested that the cell apoptotic process was initiated by the heated environment, resulted in the altered organizational structure and affected the function of blood vessels.

The results of the present study show that a high temperature was harmful to the body, where particularly in the case of lack of food and water, the heat exposure elevated blood pressure, increased arterial reactivity, which was related to the elevated production of MDA, the impaired production of SOD, and the increase of cell apoptosis. These findings are useful in gaining a better understanding of the influence of dehydrated heat exposure on vascular function, and provide certain theoretical and experimental guidance for protection under high temperature.

\section{Acknowledgements}

The present study was supported by the National Natural Science Foundation of China (no. 81560052).

\section{References}

1. Crandall CG and González-Alonso J: Cardiovascular function in the heat-stressed human. Acta Physiol (Oxf) 199: 407-423, 2010.

2. Green RS, Basu R, Malig B, Broadwin R, Kim JJ and Ostro B: The effect of temperature on hospital admissions in nine California counties. Int J Public Health 55: 113-121, 2010. 
3. Knowlton K, Rotkin-Ellman M, King G, Margolis HG, Smith D, Solomon G, Trent R and English P: The 2006 California heat wave: Impacts on hospitalizations and emergency department visits. Environ Health Perspect 117: 61-67, 2009.

4. Cheuvront SN, Kenefick RW, Montain SJ and Sawka MN Mechanisms of aerobic performance impairment with heat stress and dehydration. J Appl Physiol 109: 1989-1995, 1985.

5. Yang M, Zhao N, Luo Y, Ding J, Nie L-H, Dong J-W and Li G-H: Effects of dehydration heat exposure on weight of stress organ and IgG, IL- 2, IL-6 and intervention of LBP in rats. Lishizhen Medicine and Materia Medica Research 7: 1761-1764, 2014.

6. Miescher E and Fortney SM: Responses to dehydration and rehydration during heat exposure in young and older men. Am J Physiol 257: R1050-R1056, 1989.

7. Manning EP and Wilson B: Dehydration in extreme temperatures while conducting stability and support operations in a combat zone. Mil Med 172: 972-976, 2007.

8. González-Alonso J: Hyperthermia impairs brain, heart and muscle function in exercising humans. Sports Med 37: 371-373, 2007.

9. Deng DF, Wang CF, Lee S, Bai S and Hung SSO: Feeding rates affect heat shock protein levels in liver of larval white sturgeon (Acipenser transmontanus). Aquaculture 287: 223-226, 2009.

10. Pathapati RM, Rajesh Kumar M, Chirra BR, et al: Acute effects of two angiotensin receptor blockers on vascular hemodynamics, arterial stiffness, and oxidative stress in patients with mild to moderate hypertension: An open label parallel group study. ISRN Vascular Medicine 2013: 1-5, 2013.

11. Cui S, Reichner JS, Mateo RB and Albina JE: Activated murine macrophages induce apoptosis in tumor cells through nitric oxide-dependent or -independent mechanisms. Cancer Res 54: 2462-2467, 1994.

12. Overton JM, Williams TD, Chambers JB and Rashotte ME: Cardiovascular and metabolic responses to fasting and thermoneutrality are conserved in obese Zucker rats. Am J Physio Regul Integr Comp Physiol 280: R1007-R1015, 2001.
13. Williams TD, Chambers JB, Henderson RP, Rashotte ME and Overton JM: Cardiovascular responses to caloric restriction and thermoneutrality in C57BL/6J mice. Am J Physiol Regul Integr Comp Physiol 282: R1459-R1467, 2002.

14. Rubini A: Effect of perfusate temperature on pulmonary vascular resistance and compliance by arterial and venous occlusion in the rat. Eur J Appl Physiol 93: 435-439, 2005.

15. Benetos A, Vasmant D, Thiéry P and Safar M: Effects of ramipril on arterial hemodynamics. J Cardiovasc Pharmacol 18 (Suppl 2): S153-S156, 1991

16. Bonetti PO, Lerman LO, Napoli C and Lerman A: Statin effects beyond lipid lowering-are they clinically relevant? Eur Heart J 24: 225-248, 2003.

17. Shan X, Zhou J, Ma T and Chai Q: Lycium barbarum polysaccharides reduce exercise-induced oxidative stress. Int J Mol Sci 12: 1081-1088, 2011.

18. Mc Clean CM, Mc Laughlin J, Burke G, Murphy MH, Trinick T, Duly E and Davison GW: The effect of acute aerobic exercise on pulse wave velocity and oxidative stress following postprandial hypertriglyceridemia in healthy men. Eur J Appl Physiol 100: 225-234, 2007.

19. Urso ML and Clarkson PM: Oxidative stress, exercise, and antioxidant supplementation. Toxicology 189: 41-54, 2003

20. Kumar S and Vaux DL: Apoptosis. A cinderella caspase takes center stage. Science 297: 1290-1291, 2002.

21. Radwan MA, El-Gendy KS and Gad AF: Oxidative stress biomarkers in the digestive gland of Theba pisana exposed to heavy metals. Arch Environ Contam Toxicol 58: 828-835, 2010.

22. Liu Y, Zheng Q, Wu H, Guo X, Li J and Hao S: The effect of rapamycin on expression ratio of $\mathrm{Bax} / \mathrm{Bcl}-2$ and the expression of activated caspase-3 in different types of tumor cells. Tumor 33: 138-145, 2013. 